\title{
\begin{tabular}{l|l|l|l|l}
\hline $\mathrm{M}$ & $\mathrm{R}$ & $\mathrm{S}$ & Internet Journal of & Nitride Semiconductor Research \\
\hline
\end{tabular}
}

Volume 2, Article 36

\section{Temperature behaviour of the yellow emission in GaN}

\author{
R. Seitz, C. Gaspar, T. Monteiro, E. Pereira \\ Departamento de Física, Universidade de Aveiro \\ M. Leroux, B. Beaumont, Pierre Gibart \\ Centre de Recherche sur l'Hetero-Epitaxie et ses Applications, CRHEA-CNRS
}

This article was received on July 16, 1997 and accepted on September 15, 1997.

\begin{abstract}
Even in good quality undoped GaN samples, as assessed by the intense excitonic emission, the yellow band is present. This band has been attributed either to a shallow donor to deep double donor pair recombination [1], to a deep donor to a shallow acceptor [2] or to a shallow donor and a deep state

[3]. However, its origin is not yet clear. We present data on time resolved spectroscopy compared with steady state results. These results indicate that there is no difference in band shape between steady state and time resolved spectra at all temperatures. However, in some samples there is an increase in intensity of the yellow band. It is concluded that besides a fast emission, due to prompt excitation of the centre, an indirect path from a trap $13.7 \mathrm{meV}$ below the shallow donor is responsible for the long component of the decay and the intensity increase. An emission with a lifetime of ca. $300 \mathrm{~ms}$ is also present with a maximum at $2.35 \mathrm{eV}$.
\end{abstract}

\section{Introduction}

Photoluminescence $(\mathrm{PL})$ measurements on nominally undoped n-type GaN bulk and epitaxial layers grown by different techniques on different substrates usually show besides the near band edge emission a broad yellow band with maximum at $2.25 \mathrm{eV} \mathrm{[1]} \mathrm{[2]} \mathrm{[3]} \mathrm{[4]} \mathrm{[5].}$

It is known that GaN and its alloys are important materials for light-emitting devices as blue-UV lasers and LEDs, short-wavelength optical detectors and high-temperature electronics. However, the presence of unwanted deep states in the band gap due to native or extrinsic defects reduces the efficiency of devices.

The yellow band has been attributed either to a shallow donor to a deep donor (acceptor) pair recombination [1] [2], to a deep double donor to a shallow acceptor [4] or to a shallow donor and a deep state [5]. The first model accounts for a binding energy of the acceptor at $\mathrm{E}_{\mathrm{A}} \approx 860 \mathrm{meV}$ [1] and a binding energy of the donor of $E_{D}>35 \mathrm{meV}$ [2]. In the second one, the donor state is located $700 \mathrm{meV}$ below the conduction band (CB) while the acceptor level is located $200 \mathrm{meV}$ above the valence band (VB). The third model agrees with a deep state near $1 \mathrm{eV}$ above the VB [5] not far from the energy of the deep acceptor of previous work [1] [2]. Currently most of the experimental evidence favours the first model.

In order to clarify the recombination mechanisms involved in the yellow band emission different techniques as photoluminescence [1] [2] [3] [4] [5], optically detected magnetic resonance (ODMR) [2] [4], time resolved ODMR [6], photocapacitance [5], deep level transient spectroscopy (DLTS) [5], cathodoluminescence [7] [8] and PL under hydrostatic pressure [9] have been used. However its origin and the nature of shallow and deep levels still lacks explanation.

As this emission band is detected in different samples grown under different conditions native defects as $V_{N}$ and $\mathrm{Ga}_{\mathrm{l}}$ are potential candidates involved in the recombination mechanism. The $\mathrm{n}$-type conductivity of 
undoped GaN is commonly attributed to these point defects [10] [11]. Also, impurities as $O$ [12] and $C$ [1] as well as complex defects like $\mathrm{V}_{\mathrm{Ga}}-\mathrm{O}$ [13] have been considered to explain the yellow luminescence

recombination. In the present work we identify a level $13.7 \mathrm{meV}$ below the shallow donor responsible for an intensity increase with temperature of the yellow band luminescence and an underlying band of long lifetime in the high energy side of the yellow band.

\section{Materials and Methods}

The samples used in this work were undoped wurtzite GaN layers grown by metal organic vapour phase epitaxy (MOVPE) on (0001) sapphire substrates at a growth temperature of $\sim 1050^{\circ} \mathrm{C}$. TrimethylGallium was used as $\mathrm{Ga}$ metalorganic and $\mathrm{NH}_{3}$ as nitrogen precursor. The layer thickness was $500 \mathrm{~nm}$ and the carrier concentration $10^{17}$ to $10^{18} \mathrm{~cm}^{-3}$ at RT. Time resolved photoluminescence (TRPL) spectra and lifetime measurements were carried out with a 1934C Spex phosphorimeter using a pulsed Xe arc lamp with a pulse width of $3 \mu$ s (tail after $10 \mu$ s accounts for $1 \%$ of initial intensity) as light source. Steady state photoluminescence (SSPL) spectra were obtained after excitation above the band gap by using a Xe arc lamp as light source in combination with a monochromator. In both cases the luminescence was dispersed by a Spex 1704 monochromator and detected by a photomultiplier. The samples were held in a cold tip of a closed cycle Air-Products He cryostat in a temperature range between $10 \mathrm{~K}$ and RT.

\section{Experimental Results and Discussion}

In figure 1 the steady state luminescence spectra of a non-intentionally doped MOVPE grown GaN sample are shown for different temperatures. There is a noticeable increase in luminescence intensity of the yellow band from $30 \mathrm{~K}$ to ca. $100 \mathrm{~K}$ (figure 1a ), followed by a steady decrease. This feature is not found in all samples, as in most the luminescence stays fairly constant for lower temperatures and becomes thermally quenched above $120 \mathrm{~K}$ [2]. The temperature dependence of the intensity of the yellow band is shown in figure 2.

When the spectra are taken in transient spectroscopy we can see that the yellow band does not change for delays after the light pulse from $80 \mu$ s to $1 \mathrm{~ms}$. However, as illustrated in figure 3 for longer delays (above 10 $\mathrm{ms}$ ) a broader band starts to appear in the high energy side of the emission (centred at 2.35eV). It has a decay of ca. $300 \mathrm{~ms}$ at $10 \mathrm{~K}$, and it is not as strongly temperature quenched as the yellow band centred at $2.25 \mathrm{eV}$. This accounts for the shift towards higher energies with temperature as seen in the steady state spectra of figure $1 \mathrm{~b}$. As this emission is well separated from the yellow band in time resolved spectroscopy it does not interfere with the following analysis of the yellow band.

The temperature dependence of the lifetimes of the yellow band is shown in figure 4 . We can observe a dominant decay of $0.2 \mathrm{~ms}$ fairly temperature independent and a second component with a value of $3 \mathrm{~ms}$ at $10 \mathrm{~K}$, that between 50 and $100 \mathrm{~K}$ is strongly reduced. The fast component is of the order of magnitude commonly found for the yellow band [6]. The change in value of the slower component (that is observed only in samples where the intensity increase occurs) is seen in the temperature region where the band presents the intensity increase.

The temperature dependence of the slower component of the decay follows an exponential law (1), from which an activation energy (Ea) of $13.7 \mathrm{meV}$ can be obtained:

$$
\mathrm{t}=\mathrm{t}_{0} \exp \left(\mathrm{E}_{\mathrm{d}} / \mathrm{kT}\right)
$$

With this value for the activation energy we can fit the temperature dependence of the intensity up to $100 \mathrm{~K}$ with the equation 2 . This is shown by the full line in figure 2 .

$$
\mathrm{I}(\mathrm{T})=\mathrm{I}_{0}+\mathrm{C} \exp \left(-\mathrm{E}_{2} / \mathrm{kT}\right)
$$

From these results we can conclude that there is a level 13.7 meV below the shallow donor that can populate the levels responsible for the yellow emission. At present we cannot identify the nature of this level. Recent reports on ODMR have been presented showing that besides the shallow donor with $\mathrm{g}=1.952$, a second resonance appears with $g=1.958$, that might be associated with the level below the shallow donor [14]. For samples which do not show the intensity increase of the yellow band with temperature, the relative intensity of the yellow band emission compared with the near band edge emission is higher. The fact that in samples with a relatively strong yellow band emission there is no noticeable increase in luminescence intensity with temperature might be attributed to the fact that the emission due to the indirect population of the excited states of the yellow band represents only a minor fraction of the total emission. This might be due to a low 
concentration of the defects that are responsible for the indirect population of the yellow band. Therefore, the process is only observed in samples where the total yellow band emission is relatively weak.

The long lived emission with maximum at $2.35 \mathrm{eV}$ is also found in other samples. It can be seen independently in samples where there is no increase in intensity of the yellow band, as for delays above $10 \mathrm{~ms}$ the $2.25 \mathrm{eV}$ band emission is relatively weak in these samples. This band is responsible for the broadening and shift to higher energies of the yellow band when the temperature increases, as it becomes less quenched than the yellow band centred at $2.25 \mathrm{eV}$.

\section{Conclusions}

A trap $13.7 \mathrm{meV}$ below the shallow donor is responsible for the intensity increase of the yellow band luminescence observed between 30 and ca.100K

Although the yellow band in GaN is a common feature of most samples, complex mechanisms are involved in this emission. An intensity increase with temperature is attributed to a level $13.7 \mathrm{meV}$ below the shallow donor from where the emission originates. Time resolved spectroscopy reveals the presence of another emission with maximum at $2.35 \mathrm{eV}$ and a decay time of ca. $300 \mathrm{~ms}$ at $10 \mathrm{~K}$. This band is responsible for the shift towards higher energies with temperature as it is less temperature quenched than the yellow band.

\section{Acknowledgments}

This work has been performed under the auspices of the Commission of the European Communities, under contract BRPR-CT96-034 (DG 12 - RSMT). Also financial support by JNICT, contract PRAXIS XXI $\mathrm{PBIC/C/CTM/1925/95}$ is acknowledged.

\section{References}

[1] T. Ogino, M. Aoki, Jpn. J. Appl. Phys. 19, 2395 (1980).

[2] D. M. Hofmann, D. Kovalev, G. Steude, B. K. Meyer, A. Hoffmann, L. Eckey, R. Heitz, T. Detchprom , H. Amano, I. Akasaki, Phys. Rev. B 52, 16702-16706 (1995).

[3] T. Monteiro, E. Pereira, M. R. Correia, C. Xavier, D. M. Hofmann, B. K. Meyer, S. Fischer, A. Cremades, J. Piqueras, J.Lumin. 72, 696-700 (1997).

[4] E. R. Glaser, T. A. Kennedy, K. Doverspike, L. B. Rowland, D. K. Gaskill , J. A. Freitas, Jr., M. Asif Khan, D. T. Olson, J. N. Kuznia , D. K. Wickenden , Phys. Rev. B 51, 13326-13336 (1995).

[5] E. Calleja, F. J. Sanchez, D. Basak, M. A. Sanchez-Garcia, E. Munoz, I. Izpura, F. Calle, J. M. G. Tijero, J. L. Sanchez- Rojas, B. Beaumont, P. Lorenzini, P. Gibart, Phys. Rev. B 55, 4689 (1997).

[6] F. K. Koschnick, J. M. Spaeth, E. R. Glaser, K. Doverspike, L. B. Rowland, D. K. Gaskill, D. K. Wickenden, Sci. Forum 196, 37-42 (1995).

[7] J Piqueras, C Xavier, T Monteiro, E Pereira, S Fischer, DM Hofmann, BK Meyer, A Cremades, Mater. Sci. Eng. B 42, 230-234 (1996).

[8] X Li, JJ Coleman, Appl. Phys. Lett. 70, 438-440 (1997).

[9] T. Suski, P. Perlin, H. Teisseyre, M. Leszczynski, I. Grzegory, J. Jun, M. Bockowski, S. Porowski, T. D. Moustakas , Appl. Phys. Lett. 67, 2188-2190 (1995).

[10] P. Boguslawski, E. L. Briggs, J. Bernholc, Phys. Rev. B 23, 17255 (1995).

[11] David W. Jenkins, John D. Dow, Phys. Rev. B 39, 3317-3329 (1989).

[12] J. C. Zolper, R. G. Wilson, S. J. Pearton , R. A. Stall , Appl. Phys. Lett. 68, 1945-1947 (1996). 
[13] J Neugebauer, CG Van de Walle, Appl. Phys. Lett. 69, 503-505 (1996).

[14]P. Gibart, presented at the European GaN Workshop 2, Valbonne, June 1997

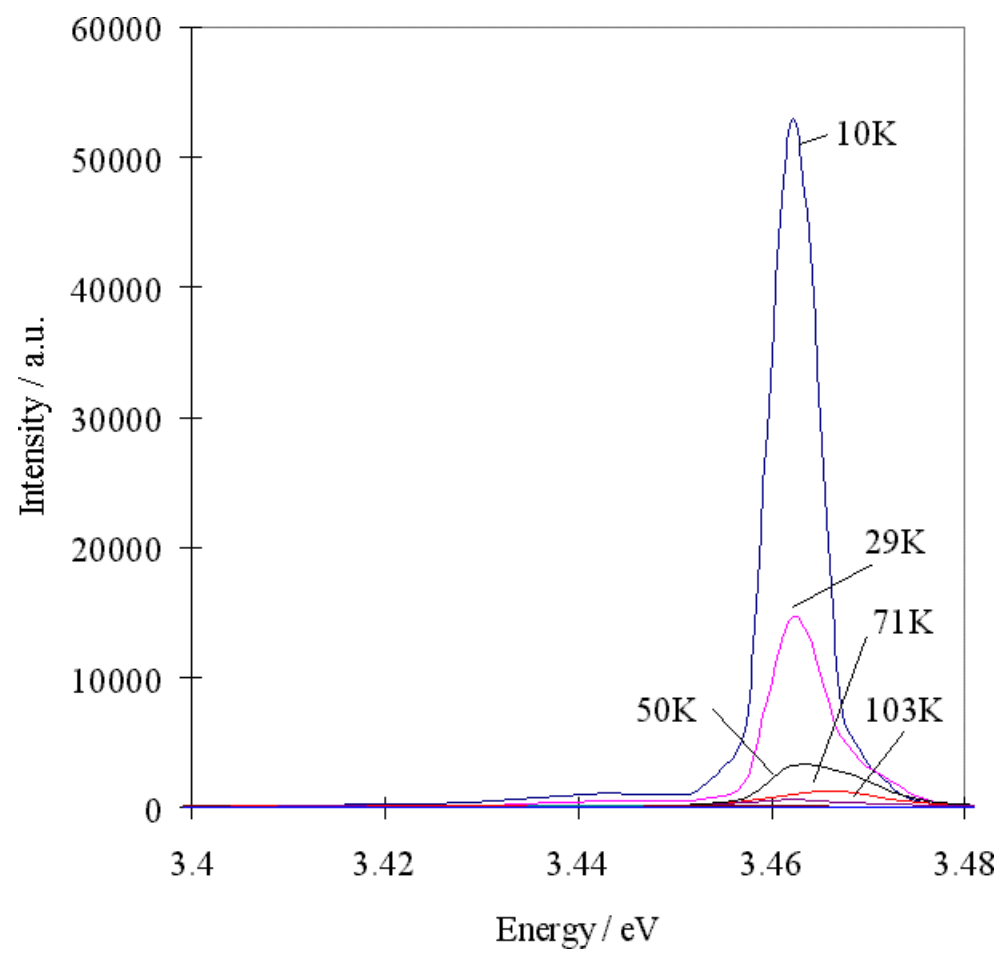

Figure 1. Steady state luminescence spectra for different temperatures.

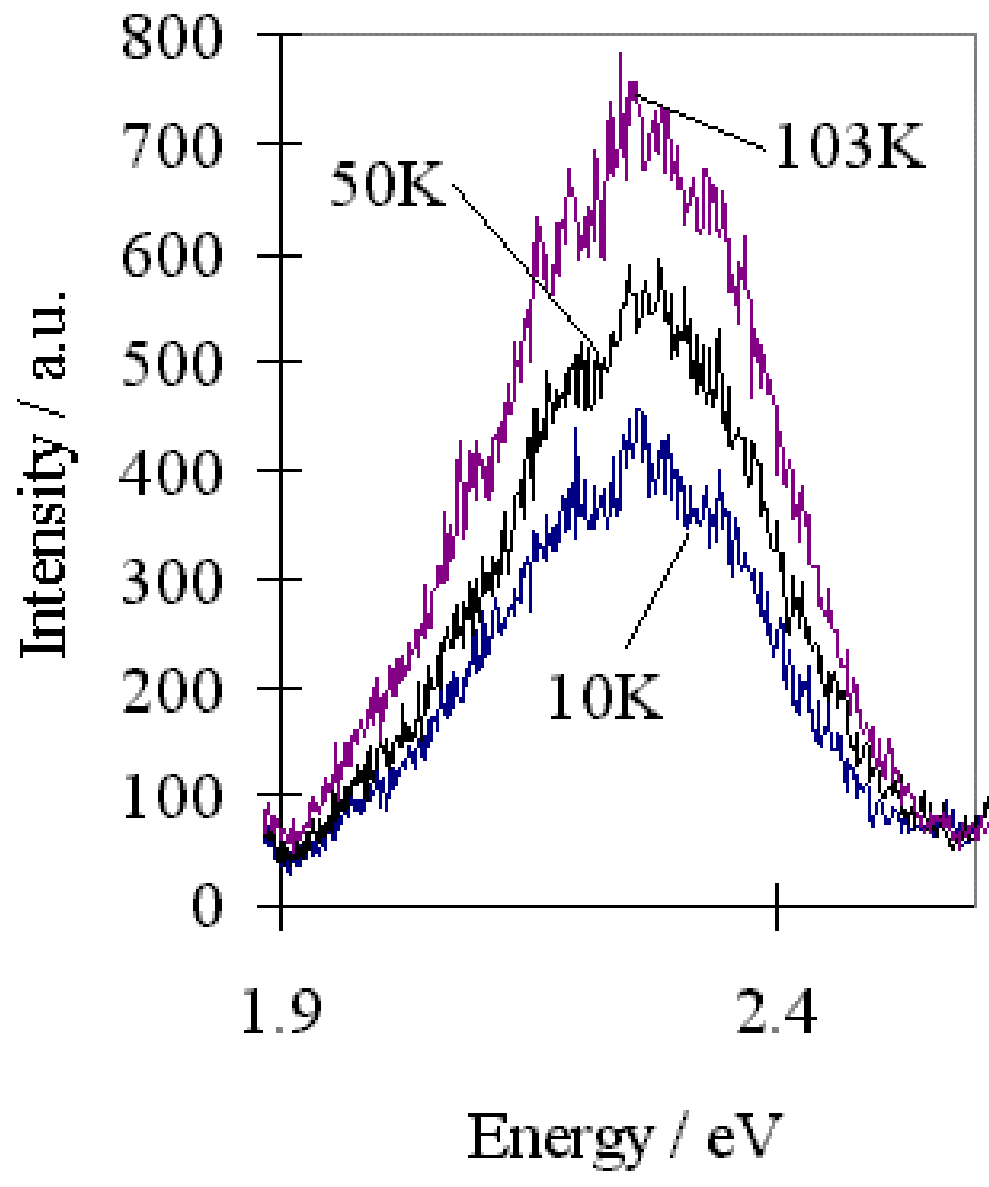

Figure 1a. Steady state luminescence spectra for different temperatures. Expanded yellow band region. 


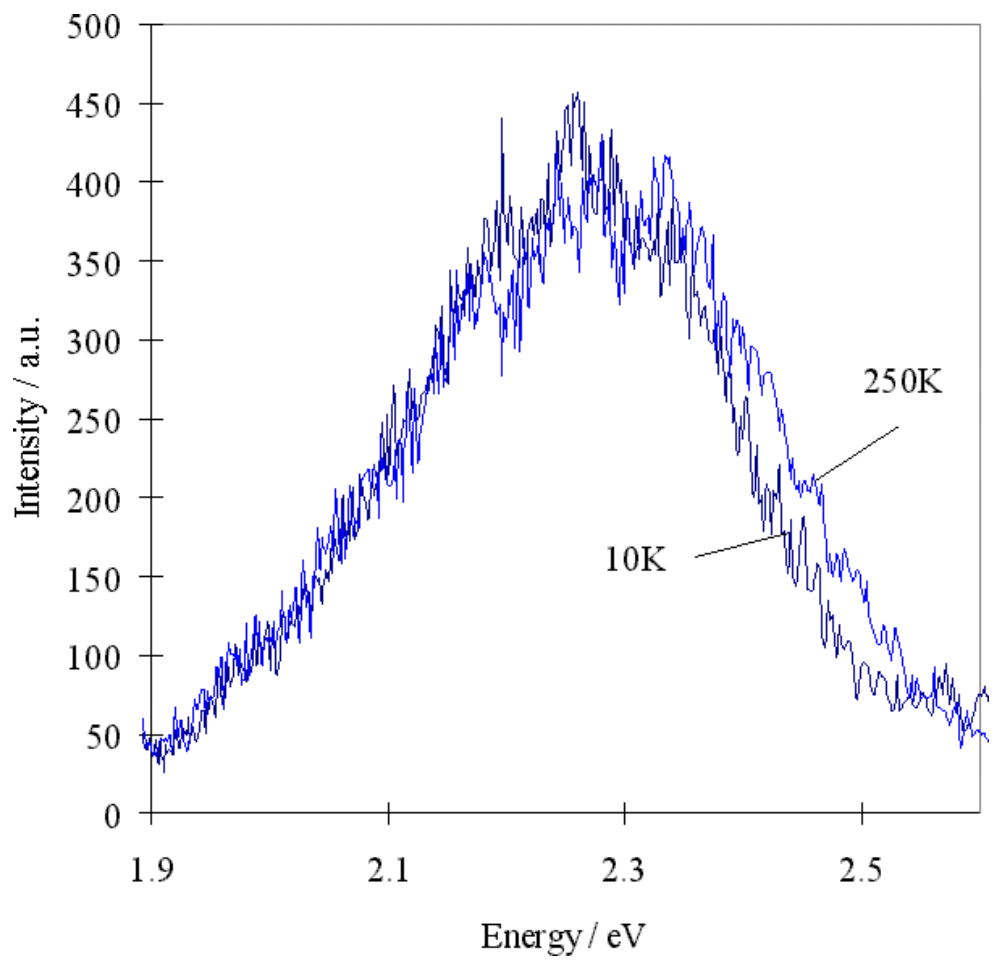

Figure 1b. Steady state luminescence spectra for different temperatures. High energy shift of the yellow band with temperature.

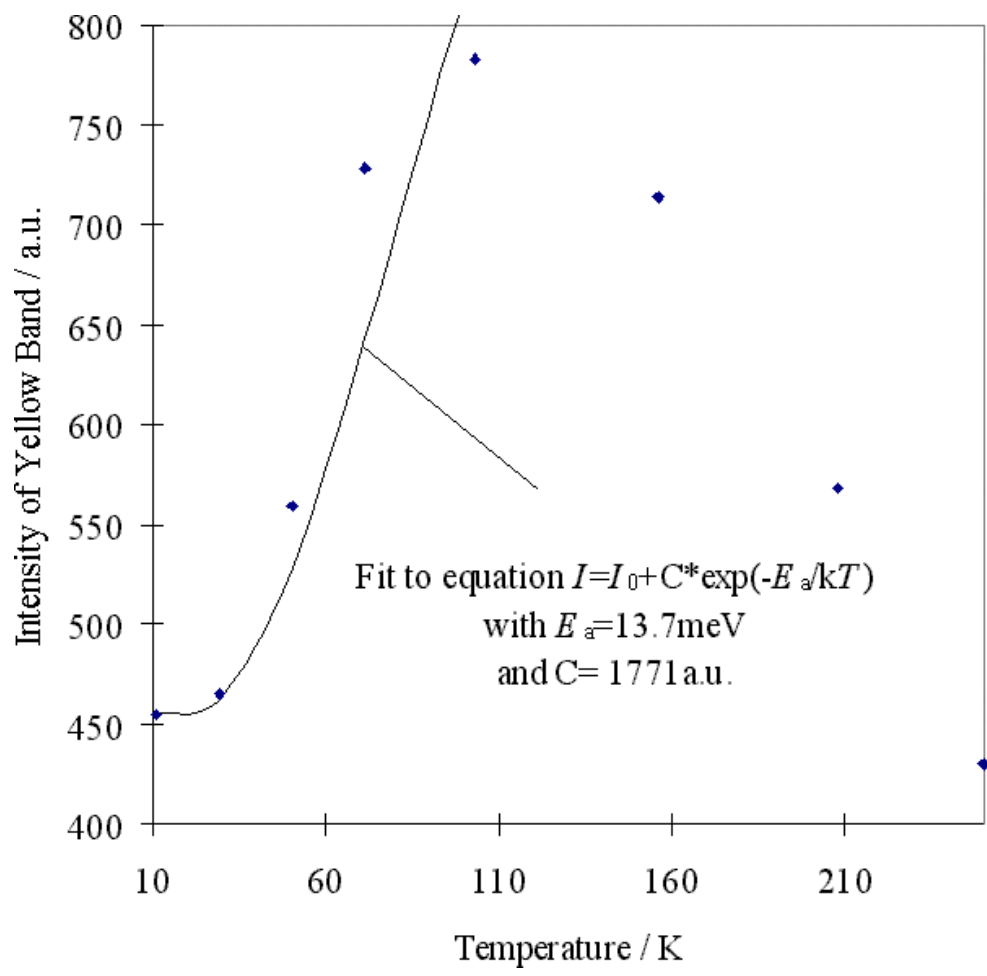

Figure 2. Temperature dependence of the yellow band intensity. Solid line: fit to equation 2 . 


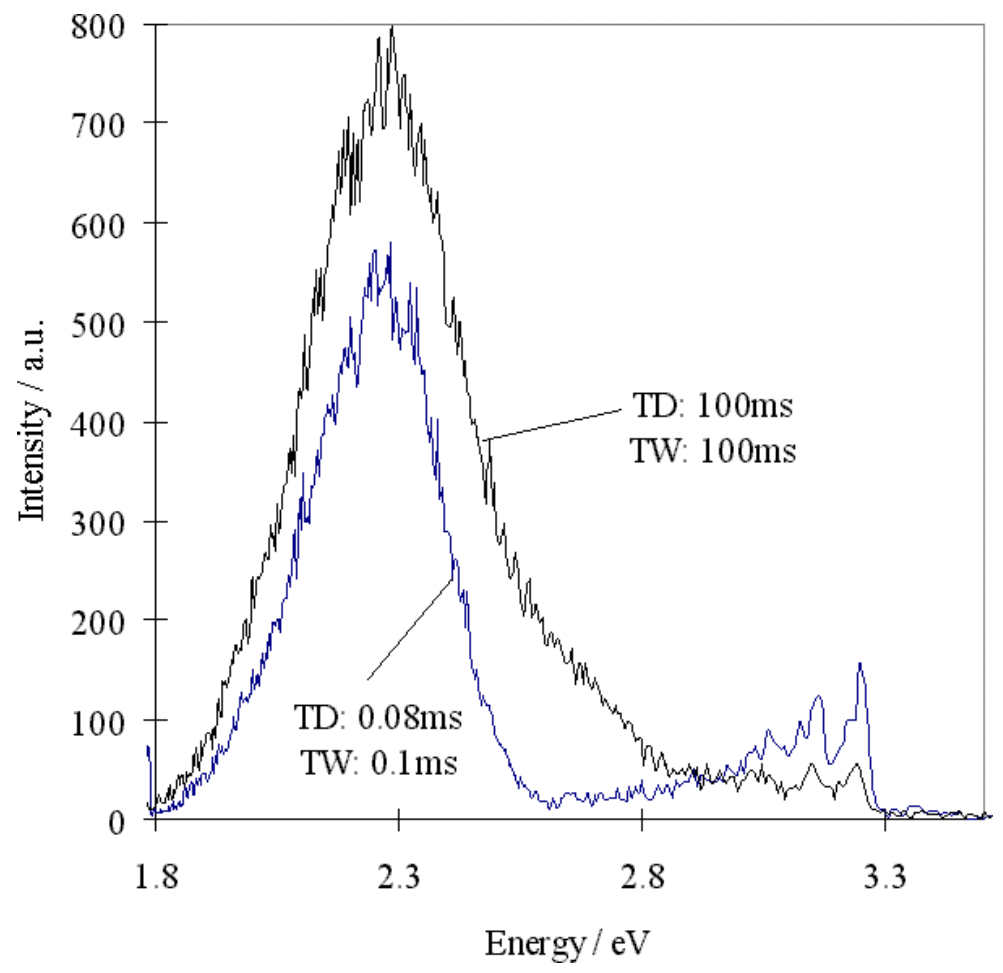

Figure 3. Time resolved spectra for different time delays (TD) and time windows (TW) at $10 \mathrm{~K}$.

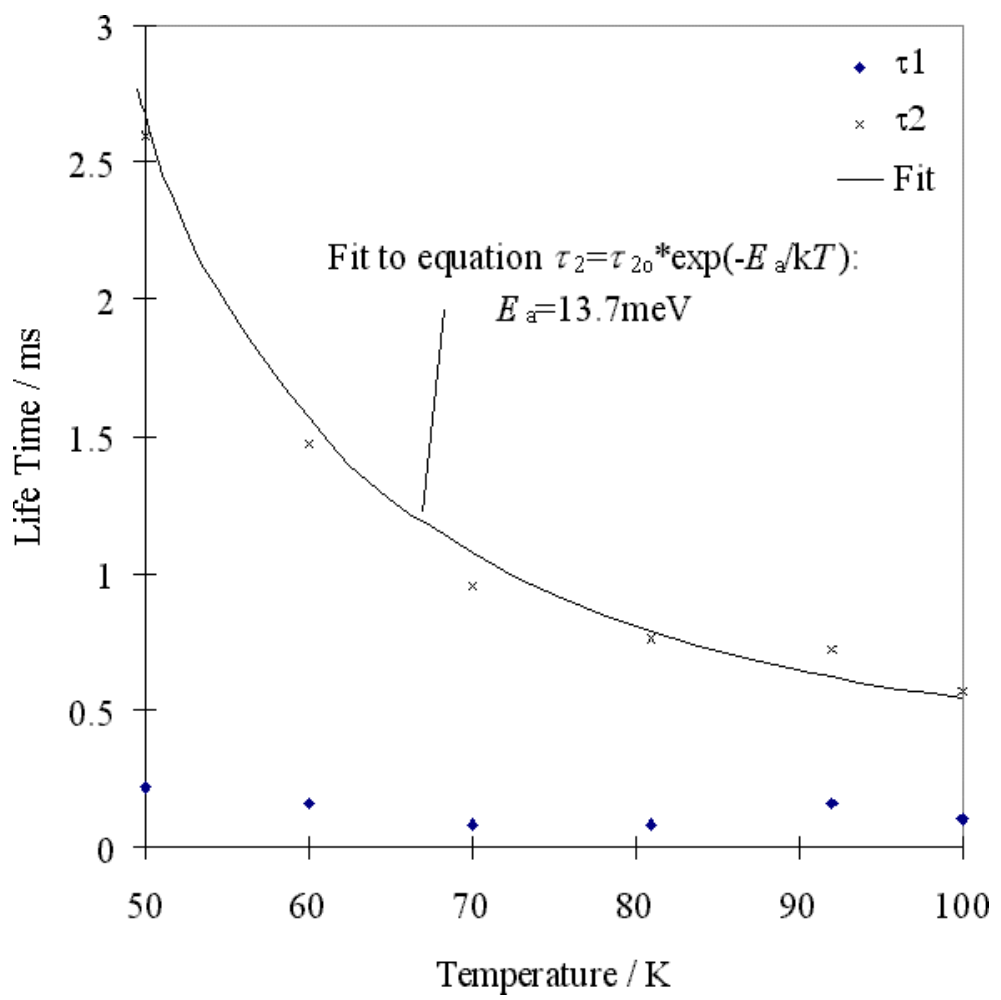

Figure 4. Temperature dependence of lifetimes. Solid line: fit of the slow decay according to equation 1 .

(C) 1997 The Materials Research Society

\begin{tabular}{|l|l|l|l|l}
\hline$M$ & $R$ & $S$ & Internet Journal of & Nitride Semiconductor Research
\end{tabular}

